\title{
Performance Enhancement of Discrete Multi-Tone Systems with a Trigonometric Transform
}

\author{
Samir Abd Elghafar ${ }^{1,2}$, Salaheldin M. Diab ${ }^{1}$, Bassiouny M. Sallam ${ }^{1}$, Moawad I. Dessouky ${ }^{1}$, \\ El-Sayed M. El-Rabaie ${ }^{1}$, Fathi E. Abd El-Samie ${ }^{1}$ \\ ${ }^{1}$ Department of Electronics and Electrical Communications, Faculty of Electronic Engineering, \\ Menoufia University, Menouf, Egypt \\ ${ }^{2}$ Department of Electrical Engineering, Jazan University, Jazan, KSA \\ Email: samir.ghaffer@yahoo.com, dr_salah_diab@yahoo.com, b_m salam@hotmail.com, \\ dr_moawad@yahoo.com, srabie1@yahoo.com, fathi_sayed@yahoo.com
}

Received October 10, 2013; revised November 10, 2013; accepted November 17, 2013

Copyright (C) 2014 Samir Abd Elghafar et al. This is an open access article distributed under the Creative Commons Attribution License, which permits unrestricted use, distribution, and reproduction in any medium, provided the original work is properly cited. In accordance of the Creative Commons Attribution License all Copyrights (C) 2014 are reserved for SCIRP and the owner of the intellectual property Samir Abd Elghafar et al. All Copyright (C) 2014 are guarded by law and by SCIRP as a guardian.

\section{ABSTRACT}

The sine transform can be used as a tool to conquer the problems of discrete multi-tone (DMT) systems to increase the bit rate. In the proposed discrete sine transform based discrete multi-tone (DST-DMT) system, we make use of the energy compaction property of the DST to reduce the channel effects on the transmitted signals. The mathematical model of the proposed DST system is presented in the paper. Simulation experiments have been carried out to test the effect of the proposed DST-DMT system. The results of these experiments show that the performance of the DST-DMT system is better than that of the traditional FFT-DMT system. The results also show that employing the proposed TEQ in the DST-DMT system can increase the bit rate by about $2.57 \mathrm{Mbps}$.

\section{KEYWORDS}

\section{DMT System; Discretesine Transform; Discrete Fourier Transform; Time Domain Equalizer}

\section{Introduction}

One of the major problems in DMT systems is the interference problem, whether it is inter-symbol interference (ISI) or intercarrier interference (ICI). The data are transmitted in DMT systems in the form of symbols. Attaching a guard period of $v$ samples to each DMT symbol eradicates the ISI, when $v \geq \mathrm{L}-1$, where $\mathrm{L}$ is the length of the channel impulse response (CIR) [1]. When the guard period is a cyclic prefix (CP), i.e. a copy of the last $v$ samples of a DMT symbol, the ICI is reduced [2]. The guard period decreases the channel throughput by a factor of $\mathrm{N} /(\mathrm{N}+v)$, where $\mathrm{N}$ is both the symbol length and the FFT length. When $v$ becomes large relative to $\mathrm{N}$, this factor reduces so that the performance loss may be unreasonable. Hence, $v$ is chosen to be relatively small compared to $\mathrm{N}$. The asymmetric digital subscriber line (ADSL) and the very high-bit-rate digital subscriber line (VDSL) standards set $v$ to N/16. In the field, however, ADSL and VDSL channel impulse responses can exceed
$\mathrm{N} / 16$ samples [3].

A solution to this problem is the channel shortening equalizer, commonly known as the TEQ. This equalizer is required to shorten the length of the effective channel. The TEQ is a finite impulse response (FIR) filter. The equalized channel, which is the cascade of the channel and the TEQ, can be modeled as a delay by $\Delta$ samples followed by an FIR filter, whose impulse response is the target impulse response (TIR) of $v+1$ samples. The TIR would fit into a target window of $v+1$ samples starting at sample index $\Delta+1$ in the shortened impulse response (SIR). The rest of the SIR would ideally be zero [4].

Different TEQ design methods optimize the FIR coefficients based on training data under different criteria. The minimum mean square error (MMSE) design minimizes the mean square error between the output of the physical path consisting of the channel and FIR filter and the output of a virtual path consisting of a transmission delay $\Delta$ and a TIR [1,5-7]. The maximum shortening 
SNR (MSSNR) method attempts to minimize the ISI in the time domain [3]. This method maximizes the ratio of the energy of the effective channel impulse response inside a target window of $v+1$ samples to that outside the target window. The minimum-ISI (Min-ISI) method generalizes the MSSNR method by weighting the ISI in the frequency domain to place the ISI in unused and more noisy sub-channels [4]. The traditional TEQ-frequency domain equalizer (FEQ) structure equalizes all sub-channels in a combined fashion, which may limit the bit rate performance. Alternative receiver architecture was proposed in [8], in which the authors suggested the transfer of the TEQ operations to the frequency domain by moving the TEQ into the FEQ. The combined TEQ-FEQ would yield a multi-tap FEQ structure, in which each sub-channel (tone) is separately equalized. Another alternative structure was proposed in [9], in which the authors proposed the transfer of the FEQ into the TEQ to yield complex-valued time-domain equalizer filter banks. Combined equalization approaches yield higher data rates than decoupled approaches for the downstream ADSL case [10].

Multi-carrier modulation (MCM) techniques including the DMT and the orthogonal frequency division multiplexing (OFDM) have been used, extensively, in many wireless networks standards, such as IEEE 802.1la, IEEE 802.16a, and wire-line digital communication systems, such as the ADSL [11-13]. All of these systems employ the complex exponential functions as orthogonal bases. Particularly, in the DMT systems, modulation by the IFFT and demodulation by the FFT create orthogonal sub-channels [14]. However, the complex exponential functions are not the only orthogonal bases that can be used to construct baseband multicarrier signals. A single set of sinusoidal functions can be used as orthogonal bases to implement the MCM scheme, and this scheme can be synthesized using the DST [12].

It is known that the DST has an excellent energy compaction property. It uses only real arithmetics. This reduces the signal processing complexity, especially, for real pulse-amplitude modulation signaling, where the DFT based processing still uses complex arithmetics and suffers from the in-phase/in-quadrature imbalance problems, which may cause appreciable performance degradations [15]. A number of researchers have recently proposed the use of the DST in MCM systems, particularly, OFDM [11-13].

In this paper, we propose the DST-DMT systems to allow high bit rate transmission. The performance of the DST-DMT system with the proposed TEQ is tested and compared with the performance of the FFT-DMT system with the TEQ given in [16] over the eight CSA. In [16], a different FIR TEQ is designed for each tone, and the FFT becomes a bank of Goertzel filters, and a single tap FEQ is also used. The rest of the paper is organized as follows. The DFT-DMT system is presented in Section 2. The proposed DST-DMT system is discussed in Section 3. The TEQ design algorithm is presented in Section 4. The simulation parameters are given in Section 5. In Section 6 , the simulation results are presented and discussed. In the last section, the general conclusions are given.

\section{The DFT-DMT System}

Let $\boldsymbol{u}_{i}$ be the $i^{\text {th }} N \times 1$ sample DMT symbol to be transmitted. The preceding and the following symbols are the $\boldsymbol{u}_{i-1}$ and $\boldsymbol{u}_{i+1}$ symbols, respectively. The transmitted signal variance is $\sigma_{s}^{2}$. Let $v$ be the length of the CP and $\boldsymbol{h}=\left[h_{0}, h_{1}, \cdots, h_{N-1}\right]^{\mathrm{T}}$ is the $N \times 1$ channel impulse response. Let $\boldsymbol{w}=\left[w_{0}, w_{1}, \cdots, w_{M-1}\right]^{\mathrm{T}}$ be the $M \times 1$ TEQ. In this paper, we assume that the TEQ sub-channel filters are of equal size $(M)$ for simplicity.

Let $\Delta$ be the transmission delay of the signal between the transmitter and the receiver and let

$$
\boldsymbol{U}_{I S I}^{\Delta}=\boldsymbol{U}_{i-1}^{\Delta}+\boldsymbol{U}_{i}^{\Delta}+\boldsymbol{U}_{i+1}^{\Delta}
$$

be the convolutional matrix of the DMT symbols $i-1$, $i$ and $i+1$. Define the $N \times(N+M-1)$ matrix $\boldsymbol{U}_{i}^{\Delta}=\left[\left(\boldsymbol{U}_{i}^{\Delta}\right)_{L}\left(\boldsymbol{U}_{i}^{\Delta}\right)_{R}\right]$, where $\left(\boldsymbol{U}_{i}^{\Lambda}\right)_{R}$ and $\left(\boldsymbol{U}_{i}^{\Delta}\right)_{L}$ are, respectively, given by:

$$
\begin{gathered}
{\left[\begin{array}{cccccc}
0 & \cdots & \cdots & \cdots & \cdots & 0 \\
u_{i}^{N-v} & \cdots & \cdots & \cdots & \cdots & 0 \\
\vdots & & \vdots & \vdots & & \vdots \\
u_{i}^{N-2} & \cdots & u_{i}^{N-v} & 0 & \cdots & 0 \\
u_{i}^{N-1} & \cdots & u_{i}^{N-v-1} & 0 & \cdots & 0 \\
\vdots & & \vdots & \vdots & & \vdots \\
u_{i}^{N-2} & \cdots & u_{i}^{0} & u_{i}^{N-1} & \cdots & u_{i}^{N-v} \\
\vdots & & \vdots & \vdots & & \vdots \\
0 & \cdots & 0 & u_{i}^{N-1} & \cdots & u_{i}^{\Delta-M-1}
\end{array}\right]} \\
{\left[\begin{array}{cccccc}
u_{i}^{\Delta} & \cdots & u_{i}^{0} & u_{i}^{N-1} & \cdots & u_{i}^{N-v} \\
u_{i}^{\Delta+1} & \cdots & \cdots & u_{i}^{0} & u_{i}^{N-1} & \cdots \\
\vdots & & & & \vdots & \vdots \\
u_{i}^{N-1} & \cdots & \cdots & \cdots & u_{i}^{0} & u_{i}^{N-1} \\
0 & u_{i}^{N-1} & \cdots & \cdots & u_{i}^{1} & u_{i}^{0} \\
\vdots & & & & \vdots & \vdots \\
0 & \cdots & \cdots & \cdots & 0 & u_{i}^{N-1} \\
\vdots & & & & \vdots & \vdots \\
0 & & & & 0 & 0
\end{array}\right]}
\end{gathered}
$$

Matrices $\boldsymbol{U}_{i+1}^{\Delta}$ and $\boldsymbol{U}_{i-1}^{\Delta}$ of size $N \times(N+M-1)$ 
are, respectively, given by:

$$
\begin{gathered}
{\left[\begin{array}{ccccccc}
- & & & 0 & & & \\
u_{i+1}^{N-v} & 0 & \cdots & \cdots & 0 & \cdots & 0 \\
\vdots & & & & & & \vdots \\
u_{i+1}^{0} & u_{i+1}^{N-1} & \cdots & u_{i+1}^{N-v} & 0 & \cdots & 0 \\
\vdots & \ddots & \ddots & & \ddots & \ddots & \vdots \\
u_{i+1}^{\Delta-v-1} & \cdots & u_{i+1}^{0} & u_{i+1}^{N-1} & \cdots & u_{i+1}^{N-v} & 0
\end{array}\right]} \\
{\left[\begin{array}{ccccccc} 
& \mid & u_{i-1}^{N-1} & u_{i-1}^{N-2} & \cdots & u_{i-1}^{v+\Delta-M+1} \\
& \mid & \vdots & & & \vdots \\
0 & \mid & 0 & u_{i-1}^{N-1} & \cdots & u_{i-1}^{N+v-M+1} \\
& \mid & \vdots & \ddots & & \vdots \\
& \mid & 0 & \cdots & 0 & u_{i-1}^{N-1} \\
& & & - & - & -
\end{array}\right]}
\end{gathered}
$$

Let $\boldsymbol{H}$ be the $(N+M-1) \times M$ convolution matrix of the CIR and TEQ given by:

$$
\left[\begin{array}{ccccc}
h_{0} & 0 & 0 & \cdots & 0 \\
h_{1} & h_{0} & 0 & \cdots & 0 \\
\vdots & & & & \vdots \\
h_{M-1} & \cdots & & \cdots & h_{0} \\
\vdots & & & & \vdots \\
h_{N-1} & \cdots & & \cdots & h_{N-1-M} \\
0 & h_{N-1} & \cdots & \cdots & h_{N-M} \\
\vdots & & & & \vdots \\
0 & 0 & 0 & \cdots & h_{N-1}
\end{array}\right]=\left[\begin{array}{c}
\left(\boldsymbol{H}_{\mathrm{u}}\right)_{\Delta \times M} \\
\left(\boldsymbol{H}_{\mathrm{c}}\right)_{v \times M} \\
\left(\boldsymbol{H}_{\mathrm{b}}\right)_{(N-v-\boldsymbol{\Delta}+M-1) \times M}
\end{array}\right](6)
$$

For the conventional DFT-DMT system, the channel input sequence $\left\{x_{n}\right\}$ at the output of the IFFT block, as shown in Figure 1, can be presented as [17], where $X_{k}$ is the encoded bit stream.

So, we can define the vector

$$
\boldsymbol{q}_{k}=\left[1, \mathrm{e}^{j 2 \pi k / N}, \cdots, \mathrm{e}^{j 2 \pi(N-1) k / N}\right]^{\mathrm{T}}
$$

such that the inner product of $\boldsymbol{q}_{k}^{\mathrm{H}}$ with an $N$-points vector gives the $k^{\text {th }}$ FFT coefficient of that vector, where $(.)^{\mathrm{H}}$ is the Hermitian conjugate transpose operator.

Let the near end crosstalk (NEXT) or additive white

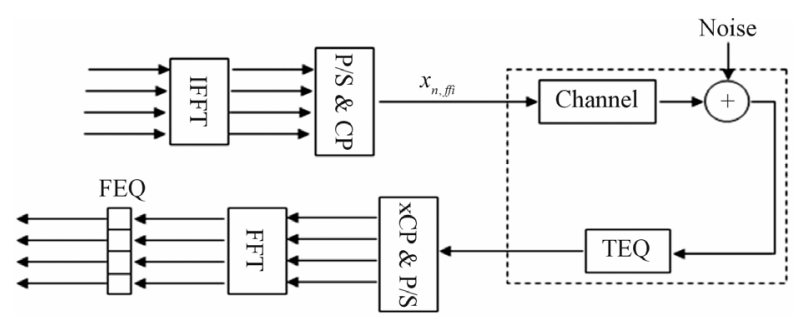

Figure 1. DFT-DMT system model. xCP means remove the CP.
Gaussian noise (AWGN) vector be:

$$
\boldsymbol{n}^{x x}=\left\{n_{-M+1}^{x x}, n_{-M+2}^{x x}, \cdots, n_{0}^{x x}, \cdots n_{N-1}^{x x}\right\}^{\mathrm{T}}
$$

Then the $(N+M-1) \times M$ AWGN or NEXT convolution matrices with the TEQ, $\boldsymbol{G}_{\mathrm{AWGN}}$ or $\boldsymbol{G}_{\mathrm{NEXT}}$ is:

$$
\begin{gathered}
\boldsymbol{G}_{x x}=\left[\begin{array}{cccc}
n_{0}^{x x} & n_{-1}^{x x} & \cdots & n_{-M+1}^{x x} \\
n_{1}^{x x} & n_{0}^{x x} & \cdots & n_{-M+2}^{x x} \\
\vdots & & & \vdots \\
n_{M-1}^{x x} & \cdots & \cdots & n_{0}^{x x} \\
\vdots & & & \vdots \\
n_{N-1}^{x x} & \cdots & \cdots & n_{N-1-M}^{x x} \\
\vdots & & & \vdots \\
n_{N+M}^{x x} & n_{N+M-1}^{x x} & \cdots & n_{N-1}^{x x}
\end{array}\right] \\
\boldsymbol{Y}_{R}^{k}(\boldsymbol{w})=\boldsymbol{q}_{k}^{\mathrm{H}}\left(\boldsymbol{U}_{I S I}^{\Delta} \boldsymbol{H}+\boldsymbol{G}_{\mathrm{AWGN}}+\boldsymbol{G}_{\mathrm{NEXT}}\right) \boldsymbol{w}, \\
k \in\{0, \cdots, N / 2-1\}
\end{gathered}
$$

The received data contains the noise due to the ISI, ICI, AWGN, NEXT, and suffers from the effects of the channel. Now, we see the dependence of the received signal on the TEQ. The ideal received signal has no noise and is formatted to fit the demodulation scheme. In DMT modulation, this means that the received symbol has minimal noise due to AWGN, NEXT, and ISI. We can design the TEQ to process the received samples to achieve this target.

Next, we will express the desired signal as a function of the TEQ taps. The desired circular convolution of the $i^{\text {th }}$ symbol and the CIR in the $k^{\text {th }}$ sub-channel, after the TEQ and FFT, can be written as:

$$
\boldsymbol{Y}_{D}^{k}(\boldsymbol{w})=\boldsymbol{q}_{k}^{\mathrm{H}}\left[\boldsymbol{U}_{i}^{\Delta}\right]_{\text {circ }} \boldsymbol{H w}, k \in\{0, \cdots, N / 2-1\}
$$

and the $N \times(N+M-1)$ circulant matrix $\left[\boldsymbol{U}_{i}^{\Delta}\right]_{\text {circ }}$ is:

$$
\left[\begin{array}{cccccc}
u_{i}^{\Delta} & \cdots & u_{i}^{0} & u_{i}^{N-1} & \cdots & u_{i}^{\Delta-M+2} \\
\vdots & & & & & \vdots \\
u_{i}^{N-1} & \cdots & u_{i}^{\Delta} & u_{i}^{\Delta-1} & \cdots & u_{i}^{N-M+1} \\
u_{i}^{0} & \cdots & u_{i}^{\Delta+1} & u_{i}^{\Delta} & \cdots & u_{i}^{N-M+2} \\
\vdots & & & & & \vdots \\
u_{i}^{\Delta-1} & \cdots & u_{i}^{0} & u_{i}^{N-1} & \cdots & u_{i}^{\Delta-M-1}
\end{array}\right]
$$

So, the received data $\boldsymbol{Y}_{R}^{k}(\boldsymbol{w})$ can be rewritten as:

$$
\boldsymbol{Y}_{R}^{k}(\boldsymbol{w})=\boldsymbol{Y}_{D}^{k}(\boldsymbol{w})+\underbrace{\left(\boldsymbol{Y}_{R}^{k}(\boldsymbol{w})-\boldsymbol{Y}_{D}^{k}(\boldsymbol{w})\right)}_{\text {ISI,ICI,AWGN,NEXT }}
$$

We then write $\operatorname{SNR}_{k}^{\text {Model }}(\boldsymbol{w})$ for all $k$ as

$$
\frac{E\left\{\left[\boldsymbol{Y}_{D}^{k}(\boldsymbol{w})\right]^{\mathrm{H}} \boldsymbol{Y}_{D}^{k}(\boldsymbol{w})\right\}}{E\left\{\left[\boldsymbol{Y}_{R}^{k}(\boldsymbol{w})-\boldsymbol{Y}_{D}^{k}(\boldsymbol{w})\right]^{\mathrm{H}}\left[\boldsymbol{Y}_{R}^{k}(\boldsymbol{w})-\boldsymbol{Y}_{D}^{k}(\boldsymbol{w})\right]\right\}}
$$


where $E[\cdot]$ is the statistical expectation operator and $[\cdot]^{\text {Model }}$ stands for model. The proposed SNR model is the ratio of the desired data, which excludes the effects of the noise including the ISI and ICI, to the difference between the received data and the desired data.

Derive $\tilde{\boldsymbol{A}}_{k}$ as:

$$
\tilde{\boldsymbol{A}}_{k}=\sigma_{s}^{2} \boldsymbol{H}^{\mathrm{T}} \boldsymbol{Q}_{k}^{\text {circ }}\left[\boldsymbol{Q}_{k}^{\text {circ }}\right]^{\mathrm{H}} \boldsymbol{H}
$$

where $\boldsymbol{Q}_{k}^{\text {cire }}$ is an $(N+M-1 \times N)$ matrix given by:

$$
\left[\begin{array}{ccccccc}
q_{k}^{N_{D}+1} & \cdots & \cdots & q_{k}^{N_{1}} & q_{0} & \cdots & q_{k}^{N_{D}} \\
q_{k}^{N_{D}+2} & \cdots & q_{k}^{N_{1}} & q_{0} & \cdots & \cdots & q_{k}^{N_{D}+1} \\
\vdots & & & & & & \vdots \\
q_{k}^{0} & q_{k}^{1} & \cdots & \cdots & \cdots & \cdots & q_{k}^{N_{1}} \\
\vdots & & & & & & \vdots \\
q_{k}^{M_{D}-3} & q_{k}^{M_{D}-2} & \cdots & q_{0} & \cdots & \cdots & q_{k}^{N-M_{D}-2}
\end{array}\right]
$$

and $N_{D}=N-\Delta, M_{D}=M+\Delta$ and $N_{1}=N-1$.

Similarly, derive $\tilde{\boldsymbol{B}}_{k}$ as,

$$
\begin{aligned}
\tilde{\boldsymbol{B}}_{k}= & 2 \sigma_{s}^{2}\left(\boldsymbol{H}_{\mathrm{u}}^{\mathrm{T}} \boldsymbol{V}_{k} \boldsymbol{V}_{k}^{\mathrm{H}} \boldsymbol{H}_{\mathrm{u}}+\boldsymbol{H}_{\mathrm{b}}^{\mathrm{T}} \boldsymbol{W}_{k} \boldsymbol{W}_{k}^{\mathrm{H}} \boldsymbol{H}_{\mathrm{b}}\right) \\
& +\boldsymbol{Q}_{k}^{\text {noise }}\left\{\sigma_{\text {AWGN }}^{2} \boldsymbol{I}+\sum \mathrm{NEXT}\right\}\left[\boldsymbol{Q}_{k}^{\text {noise }}\right]^{\mathrm{H}}
\end{aligned}
$$

where $\sigma_{\mathrm{AWGN}}^{2}$ is the noise variance, which is measured by the power of the noise with respect to $100 \Omega$, $\sum$ NEXT is the toeplitz variance matrix of the NEXT and $\boldsymbol{I}$ is the $M \times M$ identity matrix. Without loss of generality, define constraint set

$\boldsymbol{J}=\left\{\boldsymbol{w}: \boldsymbol{w}^{\mathrm{T}} \boldsymbol{w}=\|\boldsymbol{w}\|^{2}=1\right\}$, so that $\tilde{\boldsymbol{B}}_{k}$ becomes independent of $\boldsymbol{w}$ over this constraint set. Matrix $\boldsymbol{Q}_{k}^{\text {noise }}$ of size $M \times(N+M-1)$ is defined as:

$$
\left[\begin{array}{ccccccc}
0 & 0 & \cdots & 0 & q_{k}^{0} & \cdots & q_{k}^{N-1} \\
0 & 0 & \cdots & q_{k}^{0} & \cdots & q_{k}^{N-1} & 0 \\
\vdots & & & & & & \vdots \\
q_{k}^{0} & \cdots & q_{k}^{N-1} & 0 & \cdots & 0 & 0
\end{array}\right]
$$

where $q_{k}^{(\cdot)}$ are members of the vector $\boldsymbol{q}_{k}$ defined by Equation (8). $\boldsymbol{V}_{k}$ is a $\boldsymbol{\Delta} \times \boldsymbol{\Delta}$ upper diagonal matrix defined as:

$$
\left[\begin{array}{ccccc}
q_{k}^{N-\Delta} & q_{k}^{N-\Delta+1} & \cdots & q_{k}^{N-2} & q_{k}^{N-1} \\
q_{k}^{N-\Delta+1} & q_{k}^{N-\Delta} & \cdots & q_{k}^{N-1} & 0 \\
\vdots & & & \vdots & \vdots \\
q_{k}^{N-1} & 0 & \cdots & 0 & 0
\end{array}\right]
$$

$\boldsymbol{W}_{k}$ is a lower diagonal

$(N-v-\Delta+M-1) \times(N-v-\Delta+M-1)$ matrix defined as:

$$
\left[\begin{array}{ccccc}
0 & 0 & \cdots & 0 & q_{k}^{0} \\
0 & 0 & \cdots & q_{k}^{0} & q_{k}^{1} \\
\vdots & & & \vdots & \vdots \\
q_{k}^{0} & q_{k}^{1} & \cdots & \cdots & q_{k}^{N-v-\Delta+M-2}
\end{array}\right]
$$

$\tilde{\boldsymbol{A}}_{k}$ and $\tilde{\boldsymbol{B}}_{k}$ are Hermitian symmetric matrices. Now (15) becomes:

$$
\operatorname{SNR}_{k}^{\text {Model }}(\boldsymbol{w})=\frac{\boldsymbol{w}^{\mathrm{T}} \tilde{\boldsymbol{A}}_{k} \boldsymbol{w}}{\boldsymbol{w}^{\mathrm{T}} \tilde{\boldsymbol{B}}_{k} \boldsymbol{w}}
$$

The $\operatorname{SNR}_{k}^{\text {Model }}(\boldsymbol{w})$ is a ratio of quadratic functions of $\boldsymbol{w}$. The SNR model becomes equivalent to the SNR that could be measured at the output of the FFT in an ADSL system, when the ISI and ICI have been removed from the received signal.

Using the SNR model, the number of bits per symbol that can be supported is:

$$
\begin{aligned}
b_{D M T}(\boldsymbol{w}) & =\sum_{k \in S} \log _{2}\left(1+\frac{\mathrm{SNR}_{k}^{\mathrm{Model}}(\boldsymbol{w})}{\Gamma}\right) \\
& =\sum_{k \in S} \underbrace{\log _{2}\left(\frac{\boldsymbol{w}^{\mathrm{T}} \boldsymbol{A}_{k} \boldsymbol{w}}{\boldsymbol{w}^{\mathrm{T}} \boldsymbol{B}_{k} \boldsymbol{w}}\right)}_{b_{k}(\boldsymbol{w})} \quad \text { Bits/symbol }
\end{aligned}
$$

where $\boldsymbol{A}_{k}=\Gamma \tilde{\boldsymbol{B}}_{k}+\tilde{\boldsymbol{A}}_{k}$ and $\boldsymbol{B}_{k}=\Gamma \tilde{\boldsymbol{B}}_{k}, k$ is the subchannel index, $\boldsymbol{S}$ is the set of the indices of the used $\tilde{N}$ sub-channels out of $N / 2+1$ sub-channels, $b_{k}(w)$ is the number of bits per data symbol in the sub-channel $k, \Gamma$ is the SNR gap, and it is a function of several factors, including modulation method, allowable probability of error, gain of any coding applied, and desired system margin.

Maximizing the number of bits allocated to a single channel, $b_{k}(\boldsymbol{w})$ involves maximizing the argument of the log function. Since the log function is a monotonically increasing function of a non-negative argument, maximizing its non-negative argument will also maximize the function. Mathematical notation for this statement is:

$$
\begin{aligned}
b_{k}^{\text {opt }} & =\max _{\boldsymbol{w}_{k}:\left\|\boldsymbol{w}_{k}\right\|^{2}=1}\left[\log \left(\frac{\boldsymbol{w}_{k}^{\mathrm{T}} \boldsymbol{A}_{k} \boldsymbol{w}_{k}}{\boldsymbol{w}_{k}^{\mathrm{T}} \boldsymbol{B}_{k} \boldsymbol{w}_{k}}\right)\right] \\
& =\log _{2}\left[\max _{\boldsymbol{w}_{k}: \mid \boldsymbol{w}_{k} \|^{2}=1}\left(\frac{\boldsymbol{w}_{k}^{\mathrm{T}} \boldsymbol{A}_{k} \boldsymbol{w}_{k}}{\boldsymbol{w}_{k}^{\mathrm{T}} \boldsymbol{B}_{k} \boldsymbol{w}_{k}}\right)\right]
\end{aligned}
$$

This is the well-known generalized eigenvalue problem [16] and the solution is the generalized eigenvector $\boldsymbol{w}_{k}^{\text {opt }}$ corresponding to the largest generalized eigenvalue $\lambda_{k}^{\text {opt }}$ of $\left(\boldsymbol{A}_{k}^{r}, \boldsymbol{B}_{k}^{r}\right)$

$$
\lambda_{k}^{\mathrm{opt}}=\frac{\left(\boldsymbol{w}_{k}^{\mathrm{opt}}\right)^{\mathrm{T}} \boldsymbol{A}_{k}^{r}\left(\boldsymbol{w}_{k}^{\mathrm{opt}}\right)}{\left(\boldsymbol{w}_{k}^{\mathrm{opt}}\right)^{\mathrm{T}} \boldsymbol{B}_{k}^{r}\left(\boldsymbol{w}_{k}^{\mathrm{opt}}\right)}=\frac{\left(\boldsymbol{w}_{k}^{\mathrm{opt}}\right)^{\mathrm{T}} \boldsymbol{A}_{k}\left(\boldsymbol{w}_{k}^{\mathrm{opt}}\right)}{\left(\boldsymbol{w}_{k}^{\mathrm{opt}}\right)^{\mathrm{T}} \boldsymbol{B}_{k}\left(\boldsymbol{w}_{k}^{\mathrm{opt}}\right)}
$$

Hence, $b_{k}^{\text {opt }}=\log _{2}\left(\lambda_{k}^{\text {opt }}\right)$

where $(\cdot)^{r}$ denotes the real part. The number of bits per symbol that can be supported in the case of the conventional DFT-DMT system with TEQ filter bank is: 


$$
b_{\mathrm{DFT}-\mathrm{DMT}}^{\mathrm{opt}}=\sum_{k \in S} \log _{2}\left[\frac{\left(\boldsymbol{w}_{k}^{\mathrm{opt}}\right)^{\mathrm{T}} \boldsymbol{A}_{k}\left(\boldsymbol{w}_{k}^{\mathrm{opt}}\right)}{\left(\boldsymbol{w}_{k}^{\mathrm{opt}}\right)^{\mathrm{T}} \boldsymbol{B}_{k}\left(\boldsymbol{w}_{k}^{\mathrm{opt}}\right)}\right]
$$

Bits/symbol.

\section{The proposed DST-DMT System}

For the proposed DST-DMT system with TEQ filter bank, the channel input sequence $\left\{x_{n}\right\}$ at the output of the IDST block, as shown in Figure 2, can be presented as [18]:

$$
\begin{aligned}
x_{n, d s t}= & \left(\frac{2}{N}\right)^{\frac{1}{2}} \cdot \sum_{k=0}^{N-1} A_{k} \cdot X_{k} \cdot \sin \left[\frac{\pi k}{2 N}(2 n+1)\right], \\
& n=0,1, \cdots, N-1
\end{aligned}
$$

where

$$
A_{k}= \begin{cases}\frac{1}{\sqrt{2}} & \text { for } k=0 \\ 1 & \text { otherwise }\end{cases}
$$

and $X_{k}$ is the encoded bit stream.

So, the defined vector in Equation (8) will be:

$$
\boldsymbol{q}_{k, D S T}=\left[\sqrt{\frac{2}{N}} \sin \left(\frac{\pi k}{2 N}\right), \sqrt{\frac{2}{N}} \sin \left(\frac{3 \pi k}{2 N}\right), \cdots, \sqrt{\frac{2}{N}} \sin \left(\frac{\pi k}{2 N}(2(N-1)+1)\right)\right]^{\mathrm{T}}
$$

such that the inner product of $\boldsymbol{q}_{k, D S T}^{\mathrm{H}}$ with an $N$-points vector gives the $k^{\text {th }}$ DST coefficient of that vector. Note that in the above equation $\sqrt{\frac{2}{N}}$ will be $\sqrt{\frac{1}{N}}$ for $k=0$.

Depending on the defined vector in Equation (29) and using the above algorithm, we can derive a new $\boldsymbol{w}_{k, D S T}^{\text {opt }}$ corresponding to the largest generalized eigenvalue $\lambda_{k, D C T}^{\text {opt }}$ of $\left(\boldsymbol{A}_{k, D S T}^{r}, \boldsymbol{B}_{k, D S T}^{r}\right)$

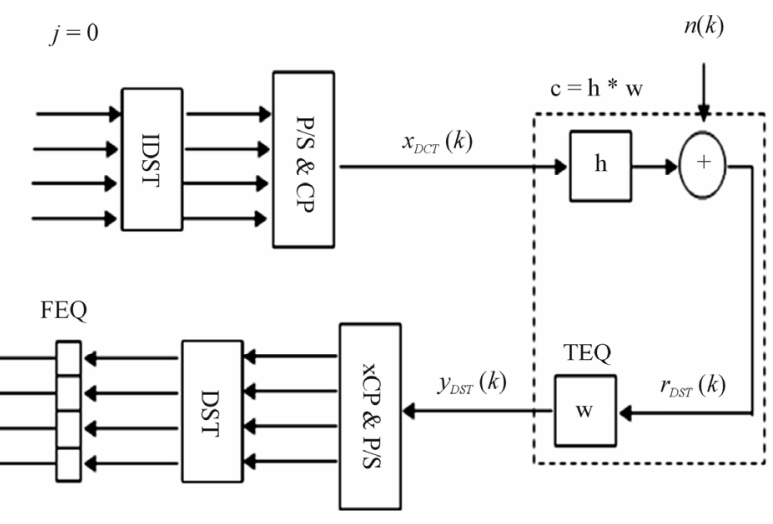

Figure 2. The proposed DST-DMT system model. xCP means remove the $\mathrm{CP}$.

$$
\begin{aligned}
\lambda_{k, D S T}^{\mathrm{opt}} & =\frac{\left(\boldsymbol{w}_{k, D S T}^{\mathrm{opt}}\right)^{\mathrm{T}} \boldsymbol{A}_{k, D S T}^{r}\left(\boldsymbol{w}_{k, D S T}^{\mathrm{opt}}\right)}{\left(\boldsymbol{w}_{k, D S T}^{\mathrm{opt}}\right)^{\mathrm{T}} \boldsymbol{B}_{k, D S T}^{r}\left(\boldsymbol{w}_{k, D S T}^{\mathrm{opt}}\right)} \\
& =\frac{\left(\boldsymbol{w}_{k, D S T}^{\mathrm{opt}}\right)^{\mathrm{T}} \boldsymbol{A}_{k, D S T}\left(\boldsymbol{w}_{k, D S T}^{\mathrm{opt}}\right)}{\left(\boldsymbol{w}_{k, D S T}^{\mathrm{opt}}\right)^{\mathrm{T}} \boldsymbol{B}_{k, D S T}\left(\boldsymbol{w}_{k, D S T}^{\mathrm{opt}}\right)}
\end{aligned}
$$

Hence,

$$
b_{k, D S T}^{\text {opt }}=\log _{2}\left(\lambda_{k, D S T}^{\text {opt }}\right)
$$

where $b_{k, D S T}^{\text {opt }}$ is the number of bits per data symbol in the sub-channel $k$. The number of bits per symbol that can be supported with the proposed DST-DMT systems implementing a TEQ filter bank is:

$$
b_{\mathrm{DST}-\mathrm{DMT}}^{\mathrm{opt}}=\sum_{k \in S} \log _{2}\left[\frac{\left(\boldsymbol{w}_{k, D S T}^{\mathrm{opt}}\right)^{\mathrm{T}} \boldsymbol{A}_{k, D S T}\left(\boldsymbol{w}_{k, D S T}^{\mathrm{opt}}\right)}{\left(\boldsymbol{w}_{k, D S T}^{\mathrm{opt}}\right)^{\mathrm{T}} \boldsymbol{B}_{k, D S T}\left(\boldsymbol{w}_{k, D S T}^{\mathrm{opt}}\right)}\right]
$$

Bits/symbol (32).

\section{TEQ Design Algorithm}

The first derivative of (23) is:

$$
\frac{\mathrm{d} b_{D M T}(\boldsymbol{w})}{\mathrm{d} \boldsymbol{w}}=\frac{2}{\ln 2} \sum_{k \in S} r_{k}(\boldsymbol{w})\left[\boldsymbol{A}_{k}^{r}-\lambda_{k}(\boldsymbol{w}) \boldsymbol{B}_{k}^{r}\right] \boldsymbol{w}
$$

where

$$
r_{k}(\boldsymbol{w})=\frac{1}{\boldsymbol{w}^{\mathrm{T}} \boldsymbol{A}_{k} \boldsymbol{w}} \text { and } \lambda_{k}(\boldsymbol{w})=\frac{\boldsymbol{w}^{\mathrm{T}} \boldsymbol{A}_{k} \boldsymbol{w}}{\boldsymbol{w}^{\mathrm{T}} \boldsymbol{B}_{k} \boldsymbol{w}}
$$

Notice that $b_{D M T}(\boldsymbol{w})=\sum_{k \in s} \log _{2}\left[\lambda_{k}(\boldsymbol{w})\right]$, thus increasing $\lambda_{k}(w)$ increases $b_{D M T}(w)$. Now we can write:

$$
\begin{aligned}
& \boldsymbol{C}_{k}(\boldsymbol{w})=r_{k}(\boldsymbol{w})\left[\boldsymbol{A}_{k}^{r}-\lambda_{k}(\boldsymbol{w}) \boldsymbol{B}_{k}^{r}\right] \text { and } \\
& \boldsymbol{C}_{k}(\boldsymbol{w}, \boldsymbol{s})=\sum_{k \in S} C_{k}(\boldsymbol{w})
\end{aligned}
$$

Let

$$
\begin{aligned}
\boldsymbol{F}(\lambda) & =\max _{\mathbf{w} \in J} \boldsymbol{w}^{\mathrm{T}} \boldsymbol{C}(\boldsymbol{w}, \boldsymbol{s}) \boldsymbol{w} \\
& =\max _{\boldsymbol{w} \in \boldsymbol{J}} \sum_{k \in \boldsymbol{s}} r_{k}\left[\boldsymbol{w}^{\mathrm{T}} \boldsymbol{A}_{k}^{r} \boldsymbol{w}-\lambda_{k} \boldsymbol{w}^{\mathrm{T}} \boldsymbol{B}_{k}^{r} \boldsymbol{w}\right]
\end{aligned}
$$

where $\lambda=\left[\cdots, \lambda_{k}, \cdots\right]^{\mathrm{T}}, k \in \boldsymbol{s}$.

Set iteration counter $i=0$, smoothing factor $\alpha=0$ and values $r_{k}$ and $\lambda_{k}$ to zero for all $k$. The algorithm proceeds as follows:

1) $r_{k}=\alpha r_{k}+(1-\alpha) \frac{1}{\boldsymbol{w}^{\mathrm{T}} \boldsymbol{A}_{k} \boldsymbol{w}}, \forall k \in \boldsymbol{s}$

2) $\lambda_{k}=\alpha \lambda_{k}+(1-\alpha) \frac{\boldsymbol{w}^{\mathrm{T}} \boldsymbol{A}_{k} \boldsymbol{w}}{\boldsymbol{w}^{\mathrm{T}} \boldsymbol{B}_{k} \boldsymbol{w}}, \forall k \in \boldsymbol{s}$

3) Compute $\boldsymbol{C}_{k}(\boldsymbol{w}, \boldsymbol{s})=\sum_{k \in \boldsymbol{s}} r_{k}\left[\boldsymbol{A}_{k}^{r}-\lambda_{k} \boldsymbol{B}_{k}^{r}\right]$ 
4) $\boldsymbol{w}_{\text {new }}=\arg \max \left\{\boldsymbol{v}^{\mathrm{T}} \boldsymbol{C}(\boldsymbol{w}, \boldsymbol{s}) \boldsymbol{v}, \boldsymbol{v} \in \boldsymbol{J}\right\}$.

5) If $\left\|\boldsymbol{w}_{\text {new }}-\boldsymbol{w}\right\|_{\infty}<\varepsilon$ or $i>i_{\max }$ then return $\boldsymbol{w}_{\mathrm{opt}}$.

6) If $b_{D M T}\left(\boldsymbol{w}_{\text {new }}\right)<b_{D M T}(\boldsymbol{w})$, set $\alpha=(1+\alpha) / 2$, else $\boldsymbol{w}_{\text {opt }}=\boldsymbol{w}_{\text {new }}$.

7) $\boldsymbol{w}=\boldsymbol{w}_{\text {new }}$.

8) $i=i+1$.

9) Go back to Step 1 and repeat.

\section{Simulation Parameters}

We use the eight CSA loops as our test channels [4]. All channel impulse responses consist of 512 samples sampled at a rate of $2.208 \mathrm{MHz}$. We add a fifth-order Chebyshevhighpass filter with cutoff frequency of $5.4 \mathrm{kHz}$ and passband ripples of $0.5 \mathrm{~dB}$ to each CSA loop to take into account the effect of the splitter at the transmitter. The DC channel (channel 0), channels $1-5$, and the Nyquist channel are not used. We model the channel noise as $-140 \mathrm{dBm}$ AWGN distributed over the bandwidth of $1.104 \mathrm{MHz}$ plus near-end-cross-talk (NEXT) noise. The NEXT noise consists of 8 ADSL disturbers as described in the ANSI T1.413 - 1995 standard [4]. The input signal power is $23 \mathrm{dBm}$ distributed equally over all used subchannels and both the FFT size, and the DST size are set to $N=512 . M=17$ and $v=32$. The coefficients of the FIR filters are obtained from the MATLAB Discrete Multitone Time-Domain Equalizer (DMTTEQ) Toolbox that was implemented by the Embedded Signal Processing Lab at the University of Texas [19].

Bandwidth optimization is applied by shutting down (not assigning any transmit power to) sub-channels with initial SNR lower than the required SNR to transmit two bits with a given SNR gap of $9.8+6-4.2=11.6 \mathrm{~dB}$. This corresponds to a system margin of $6 \mathrm{~dB}$ and a coding gain of $4.2 \mathrm{~dB}$. We are not using any bit loading algorithm, so all bit rate results are calculated from the SNR distribution after the TEQ filter bank is placed into the system. We assume that the power allocation is constant over all used sub-channels and that it is not changed after the TEQ filter bank is placed in the system.

\section{Simulation Results}

We present simulation results to analyze and compare the performance of the proposed DST-DMT system with TEQ filter bank with the conventional DFT-DMT system with a TEQ. Figure 3 compares the SNR achieved with the proposed DST-DMT system and the conventional DFT-DMT system for CSA loop 1 with $M=17, N=512$, $v=32$, input power $=23 \mathrm{dBm}$, AWGN power $=-140$ $\mathrm{dBm} / \mathrm{Hz}$, and NEXT modeled as 8 ADSL disturbers. The figure gives insight into why the performance of the proposed DST-DMT system with a TEQ filter bank outperforms the conventional DFT-DMT system with a
TEQ filter bank. The proposed system has a flat magnitude response over most of the spectrum except at the positions of the highest ISI, while for the conventional system, the SNR decreases as the frequency increases, as displayed in Figures 3-5 for CSA loops 1, 4 and 8, respectively.

Figure 6 shows the bit allocation to each sub-channelfor the conventional DFT-DMT system with a TEQ filter bank as compared to that of the proposed DSTDMT system with a TEQ filter bank for CSA loop 1 with $M=17, N=512, v=32$, input power $=23 \mathrm{dBm}$, AWGN power $=-140 \mathrm{dBm} / \mathrm{Hz}$, and NEXT modeled as $8 \mathrm{ADSL}$ disturbers. The proposed system achieves a higher bit allocation for each sub-channel over most of the spectrum except at the positions of the highest ISI, because each sub-channel carries different numbers of bits depending on its SNR. The number of bits assigned to each sub-channel in the case of the conventional system de-

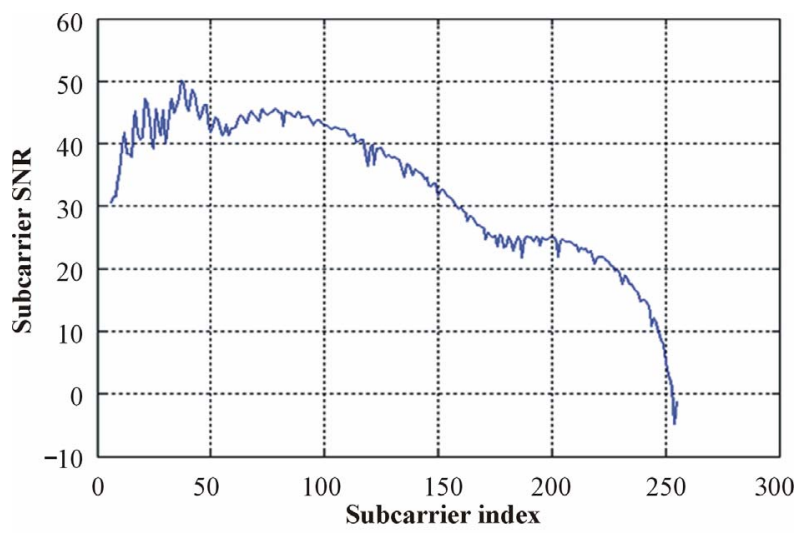

(a)

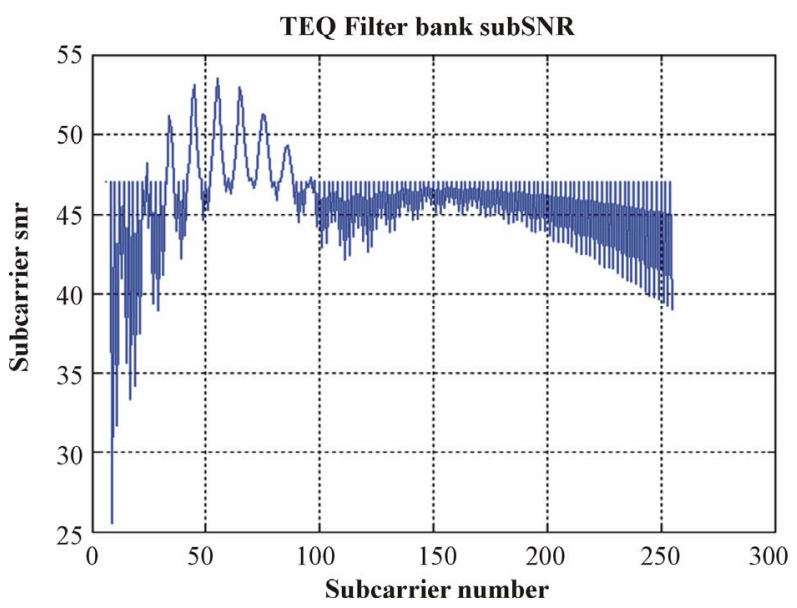

(b)

Figure 3. SNR achieved using the proposed system and the conventional system for CSA loop 1 with $M=17, N=512, v$ $=32$, input power $=23 \mathrm{dBm}$, AWGN power $=-140 \mathrm{dBm} / \mathrm{Hz}$, and NEXT is modeled as 8 ADSL disturbers. (a) The conventional DFT-DMT system with TEQ filter bank; (b) The proposed DST-DMT system with TEQ filter bank. 


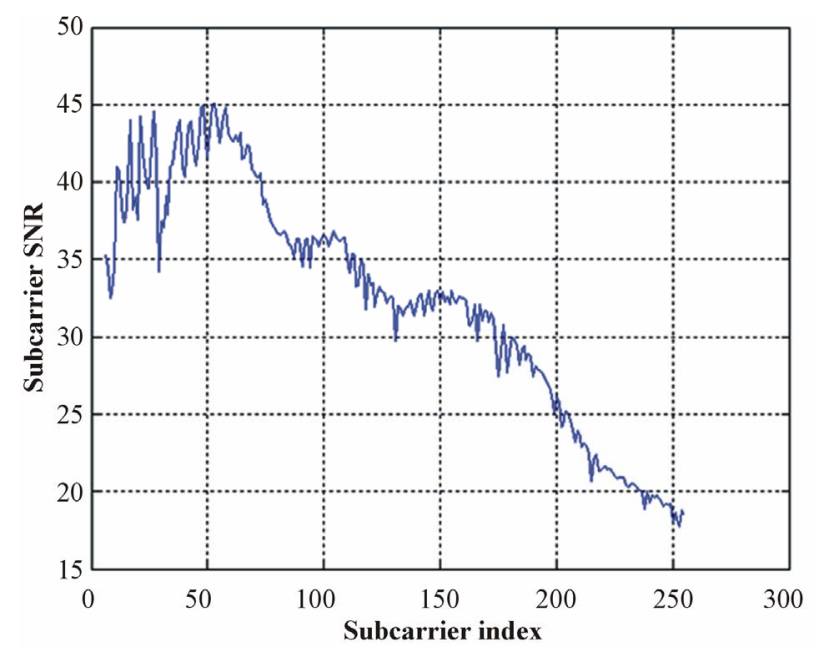

(a)

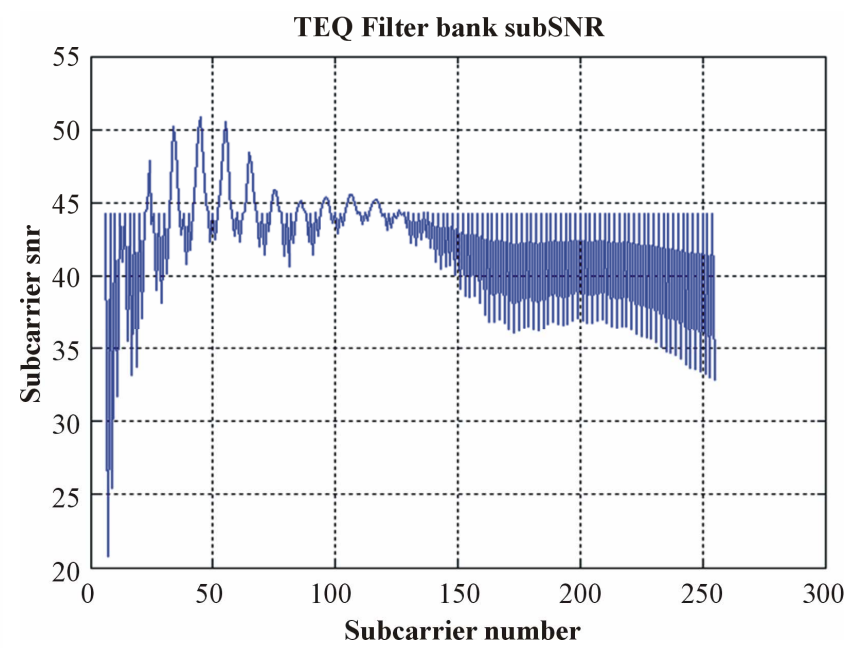

(b)

Figure 4. SNR achieved using the proposed system and the conventional system for CSA loop 4 with $M=17, N=512, v=32$, input power $=23 \mathrm{dBm}$, AWGN power $=-140 \mathrm{dBm} / \mathrm{Hz}$, and NEXT modeled as 8 ADSL disturbers. (a) Conventional DFTDMT system with TEQ filter bank; (b) The proposed DST-DMT system with a TEQ filter bank.

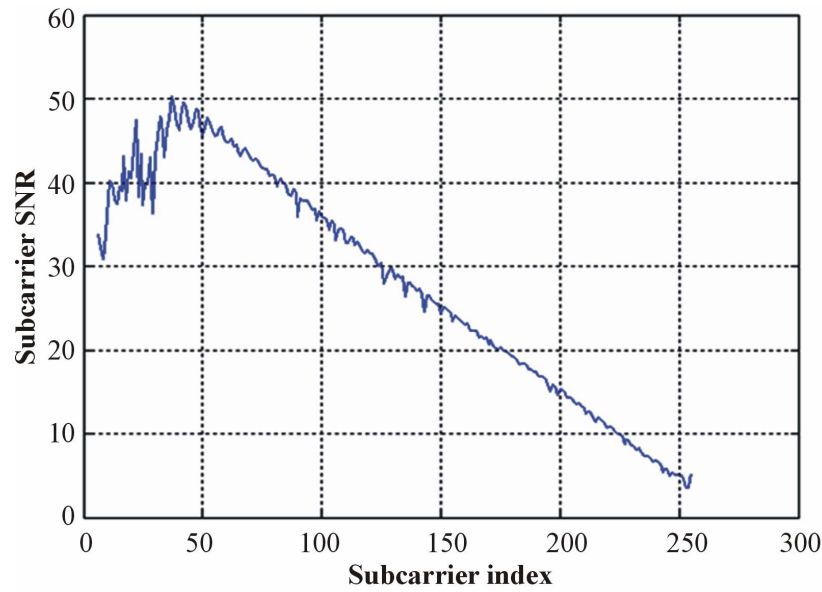

(a)

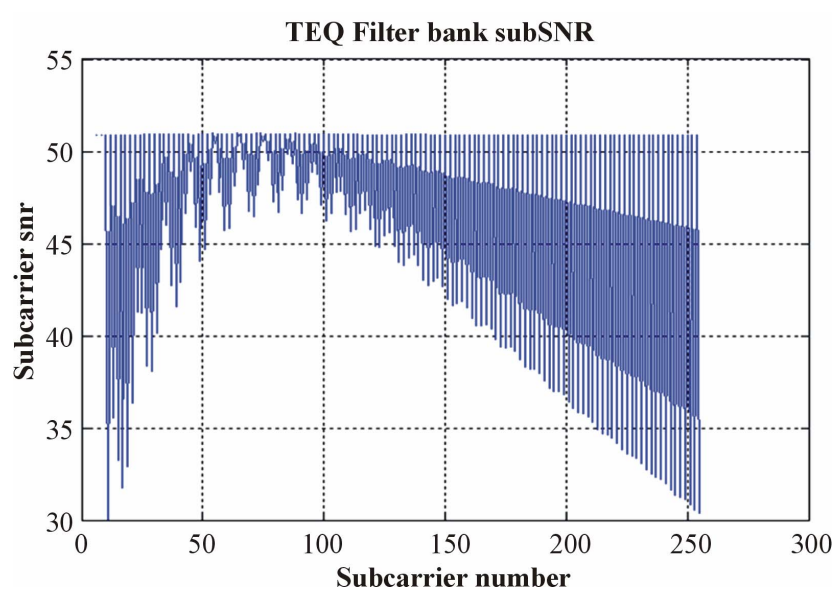

(b)

Figure 5. SNR achieved using the proposed system and the conventional system for CSA loop 8 with $M=17, N=512, v=32$, input power $=23 \mathrm{dBm}$, AWGN power $=-140 \mathrm{dBm} / \mathrm{Hz}$, and NEXT modeled as 8 ADSL disturbers. (a) Conventional DFTDMT system with a TEQ filter bank; (b) the proposed DST-DMT with a TEQ filter bank.

creases as the frequency increases, as displayed in Figures 6-8 for CSA loops 1,4 and 8 , respectively.

Table 1 lists the data rates achieved with the proposed DST-DMT system with TEQ filter bank for the CIR including CSA loops 1-8, as well as that of the conventional DFT-DMT system. The proposed DST-DMT system achieves higher data rates for each CIR than the conventional DFT-DMT system in the range of $(2.899-5.369)$ Mbps.

\section{Conclusions}

In this paper, we proposed a new TEQ filter bank along with the use of the DST in order to achieve higher bit rates in DMT systems. Simulations experiments have shown that the proposed DST-DMT system with TEQ filter bank provides a better performance than the conventional DFT-DMT system. The results indicate that the proposed DST-DMT system achieves a higher SNR in each sub-channel (over most of the spectrum except at the positions of the highest ISI) than the conventional DFT-DMT system over the eight CSA loops. It was also concluded that the proposed DST-DMT system with TEQ filter bank achieves higher bit rates than the conventional DFT-DMT system. We can say that the major advantage of the DST-DMT system with TEQ filter bank is in the energy compaction property of the DST. This property leaves most of the samples at the end of each symbol close to zero, which reduces the ISI, dramatical- 


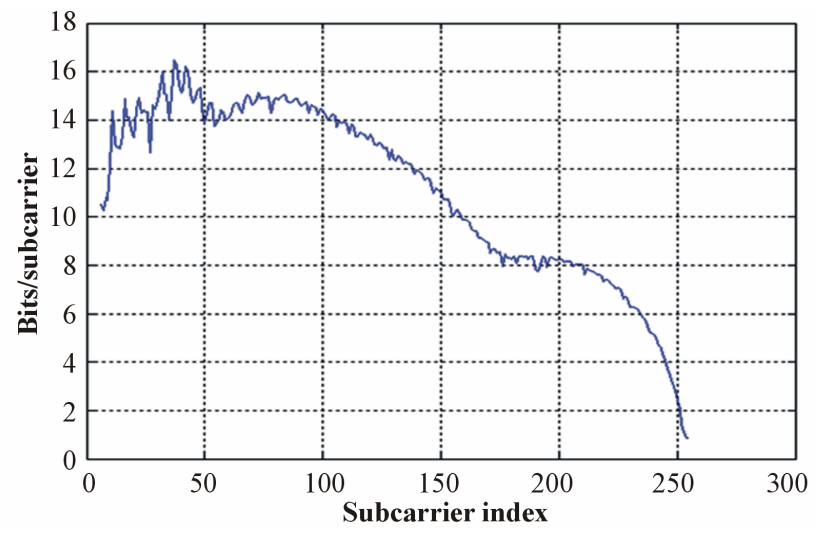

(a)

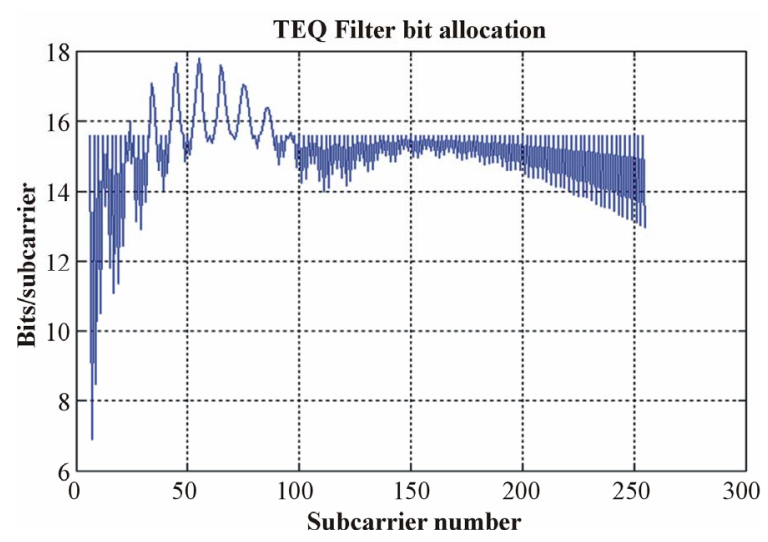

(b)

Figure 6. Bit allocation to each sub-channel using the proposed system and the conventional system for CSA loop 1 with $M=$ $17, N=512, v=32$, input power $=23 \mathrm{dBm}$, AWGN power $=-140 \mathrm{dBm} / \mathrm{Hz}$, and NEXT modeled as 8 ADSL disturbers. (a) Conventional DFT-DMT system with a TEQ filter bank; (b) The proposed DST-DMT system with a TEQ filter bank.

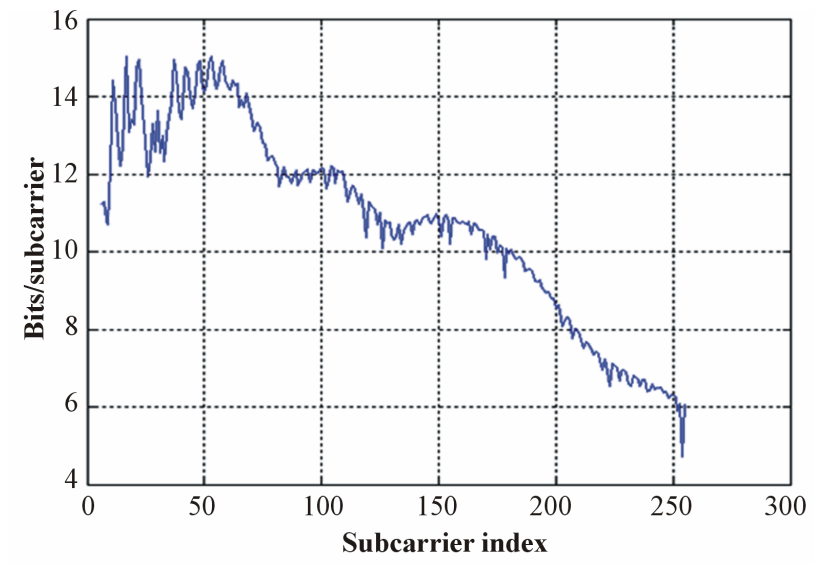

(a)

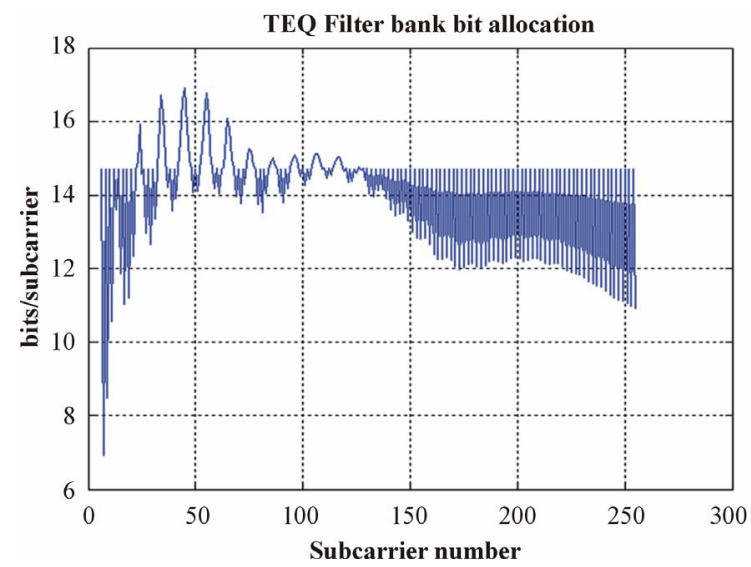

(b)

Figure 7. Bit allocation to each sub-channel achieved using the proposed system and the conventional system for CSA loop 4 with $M=17, N=512, v=32$, input power $=23 \mathrm{dBm}$, AWGN power $=-140 \mathrm{dBm} / \mathrm{Hz}$, and NEXT modeled as 8 ADSL disturbers. (a) Conventional DFT-DMT system with a TEQ filter bank. (b) The proposed DST-DMT system with a TEQ filter bank.

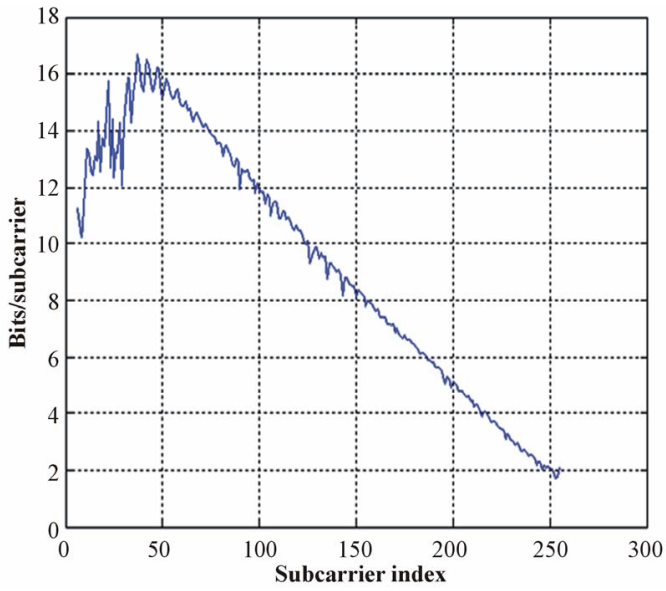

(a)

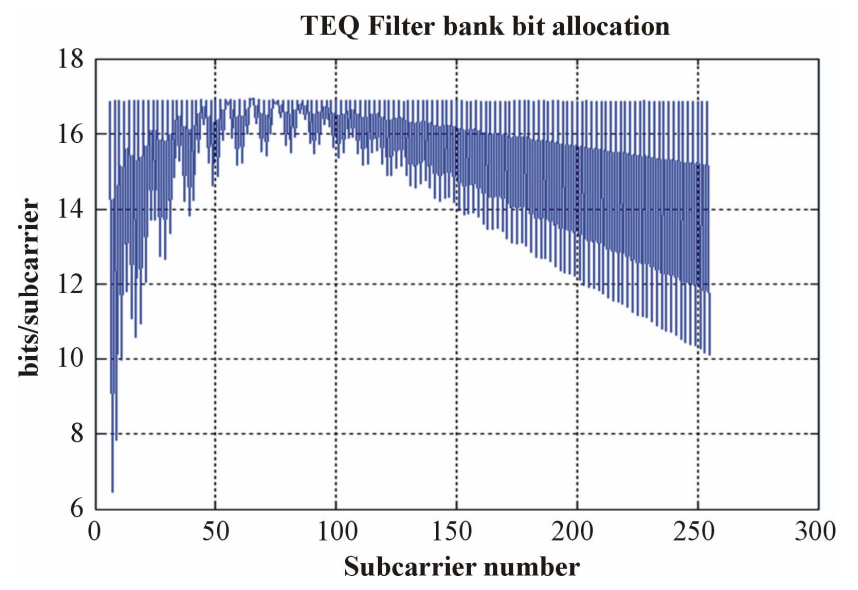

(b)

Figure 8. Bit allocation to each sub-channel achieved using the proposed system and the conventional system for CSA loop 4 with $M=17, N=512, v=32$, input power $=23 \mathrm{dBm}$, AWGN power $=-140 \mathrm{dBm} / \mathrm{Hz}$, and NEXT modeled as 8 ADSL disturbers. (a) Conventional DFT-DMT system with a TEQ filter bank; (b) The proposed DST-DMT system with a TEQ filter bank. 
Table 1. The achievable bit rates in Mbps for the proposed DST-DMT system with TEQ filter bank and the conventional DFT-DMT system for the CIR involving standard CSA loops 1-8 with $M=17, N=512, v=32$, input power $=$ $23 \mathrm{dBm}$, AWGN power $=-140 \mathrm{dBm} / \mathrm{Hz}$, and NEXT modeled as 8 ADSL disturbers.

\begin{tabular}{ccccc}
\hline & \multicolumn{2}{c}{ Bit rate (Mbps) of the } & \multicolumn{2}{c}{$\begin{array}{c}\text { Bit rate (Mbps) of the } \\
\text { Loop }\end{array}$} \\
\cline { 2 - 5 } & conventional DFT-DMT system & $\begin{array}{c}\text { MSSN } \\
\text { proposed DST-DMT system } \\
\text { with TEQ filter bank }\end{array}$ \\
\hline 1 & 5.48 & 8.592 & 11.265 & 15.078 \\
2 & 7.593 & 9.76 & 12.285 & 15.255 \\
3 & 6.933 & 8.23 & 10.963 & 15.428 \\
4 & 5.17 & 8.2 & 10.93 & 13.829 \\
5 & 6.514 & 8.64 & 11.24 & 15.352 \\
6 & 6.584 & 7.96 & 10.692 & 14.992 \\
7 & 5.728 & 7.985 & 10.359 & 14.749 \\
8 & 5.29 & 7.045 & 9.511 & 14.88 \\
\hline
\end{tabular}

ly, leading to a great performance enhancement.

\section{REFERENCES}

[1] I. Lee, J. S. Chow and J. M. Cioffi, "Performance Evaluation of a Fast Computation Algorithm for the DMT in High-Speed Subscriber Loop," IEEE Journal on Selected Areas in Communications, Vol. 13, No. 9, 1995, pp. 1564-1570. http://dx.doi.org/10.1109/49.475530

[2] K. Vanble, M. Moonen and G. Leus, "Linear and Decision-Feedback Per Tone Equalization for DMT-Based Transmission Over IIR Channels," IEEE Transactions Signal Processing, Vol. 54, No. 1, 2006, pp. 258-273.

[3] G. Arslan, B. Lu, L. D. Clark and B. L. Evans, "Iterative Refinement Methods for Time-Domain Equalizer Design," EURASIP Journal on Applied Signal Processing, Vol. 2006, 2005, pp. 1-12. http://dx.doi.org/10.1155/ASP/2006/43154

[4] G. Arslan, B. L. Evans and S. Kiaei, "Equalization for Discrete Multitone Transceivers to Maximize Bit Rate," IEEE Transactions Signal Processing, Vol. 49, No. 12, 2001, pp. 3123-3135. http://dx.doi.org/10.1109/78.969519

[5] J. S. Chow and J. M. Cioffi, "A Cost-Effective Maximum Likelihood Receiver for Multicarrier Systems," Proceedings of the IEEE International Conference on Communications, Chicago, 1992, pp. 948-952.

[6] J. S. Chow, J. M. Cioffi and J. A. Bingham, "Equalizer Training Algorithms for Multicarrier Modulation Systems," Proceedings of the IEEE International Conference on Communications, Geneva, May 1993, pp. 761-765.

[7] N. Al-Dhahir and J. M. Cioffi, "Efficiently Computed Reduced-Parameter Input-Aided MMSE Equalizers for ML Detection: A Unified Approach," IEEE Transactions on
Information Theory, Vol. 42, No. 3, 1996, pp. 903-915.

[8] K. Van Acker, G. Leus, M. Moonen, O. van de Wiel and T. Pollet, "Per Tone Equalization for DMT-Based Systems," IEEE Transactions on Communications, Vol. 49, No. 1, 2001, pp. 109-119. http://dx.doi.org/10.1109/26.898255

[9] M. Ding, Z. Shen and B. L. Evans, “An Achievable Performance Upper Bound for Discrete Multi Tone Equalization," Proceedings of IEEE Global Telecommunications Conference, Dallas, November-December 2004, pp. 2297-2301.

[10] R. K. Martin, K. Vanbleu, M. Ding, et al., "Unification and Evaluation of Equalization Structures and Design Algorithms for Discrete Multi-Tone Modulation Systems," IEEE Transactions Signal Processing, Vol. 53, No. 10, 2005, pp. 3880-3894.

[11] A. Rushdi and J. Tuqan, "PAPR Reduction in Trigonometric-Based OFDM Systems," IEEE Signals, Systems and Computers, Conference Record of the 41th Asilomar Conference, 4-7 November 2007, Pacific Grove, pp. 1747-1751.

[12] P. Tan, and N. C. Beaulieu, "A Comparison of DCTBased OFDM and DFT-Based OFDM in Frequency Offset and Fading Channels," IEEE Transactions on Communications, Vol. 54, No. 11, 2006, pp. 2113-2125. http://dx.doi.org/10.1109/TCOMM.2006.884852

[13] P. Tan and N. C. Beaulieu, "An Improved DCT-Based OFDM Data Transmission Scheme," IEEE 16th International Symposium on Personal, Indoor and Mobile Radio Communications, 11-14 September 2005, pp. 745-749.

[14] E. Khan and C. Heneghan, "Iterative Refinement Methods for Time-Domain Equalizer Design," EURASIP Journal on Applied Signal Processing, Vol. 2006, pp. 1-12, Article ID: 43154.

[15] P. Tan and N. C. Beaulieu, "A Comparison of DCTBased OFDM and DFT-Based OFDM in Frequency Offset and Fading Channels," IEEE Transactions on Communications, Vol. 54, No. 11, 2006, pp. 2113-2125. http://dx.doi.org/10.1109/TCOMM.2006.884852

[16] M. Milosevic, L. F. C. Pessoa, B. L. Evans and R. Baldick, "DMT Bit Rate Maximization with Optimal Time Domain Equalizer Filter Bank Architecture," Proceedings of the IEEE Asilomar Conference on Signals, Systems, and Computers, November 2002, Vol. 1, pp. 377-382.

[17] A.-Y. Wu and T.-S. Chan, "Cost-Efficient Parallel Lattice VLSI Architecture for the IFFT/FFT in DMT Transceiver Technology," Proceedings of the 1998 IEEE International Conference on Acoustics, Speech and Signal Processing, 1998, Vol. 6, pp. 3517-3520.

[18] A. B. Watson, "Image Compression Using the Discrete Cosine Transform," Mathematica Journal, Vol. 4, No. 1, 1994, pp. 81-88.

[19] "MATLAB Discrete Multitone Time-Domain Equalizer (DMTTEQ) Toolbox," University of Texas, Austin. http://www.ece.utexas.edu/ bevans/projects/adsl/dmtteq/ dmtteq.html 